\title{
OGLE Cepheids and RR Lyrae stars in the Milky Way
}

\author{
Andrzej Udalski ${ }^{1, \star}$ on behalf of the OGLE survey team \\ ${ }^{1}$ Warsaw University Observatory, Al. Ujazdowskie 4, 00-478 Warszawa, Poland
}

\begin{abstract}
We present new large samples of Galactic Cepheids and RR Lyrae stars from the OGLE Galaxy Variability Survey.
\end{abstract}

\section{Introduction}

The Optical Gravitational Lensing Experiment (OGLE) is one of the largest sky variability surveys. In its fourth phase it regularly monitors over a billion stars. The main OGLE observing targets are the densest stellar regions of the sky: the Galactic center and Magellanic Clouds ([10]).

One of the most important outcome of the project is the OGLE Collection of Variable Stars (OCVS). It currently consists of almost a million well characterized periodic variable stars of many different types ([9]). The OCVS contains a full variety of pulsating stars from the main astrophysical "laboratories" like the Magellanic System and the Galactic bulge. For example, the current sample of RR Lyrae stars counts over 38000 objects in the Galactic bulge and over 45000 stars in the Magellanic System (Magellanic Clouds and Magellanic Bridge, $[6,8]$ ). The number of Cepheids in the Magellanic System reaches 10000 pulsators ([7]). The OGLE samples are very pure and complete. They were used for many interesting projects related, among others, to investigations of the structure of the Galactic center ([4]) and Magellanic System ([1, 2]).

In 2013 a new OGLE long-term survey - OGLE Galaxy Variability Survey (OGLE GVS) - was initiated in the frame of the OGLE-IV phase. An area of about 2000 square degrees in the sky is regularly monitored. The survey covers a strip of \pm 3 degree width along the Galactic plane for all Galactic longitudes accessible from the OGLE observing site - Las Campanas Observatory, Chile. Additionally, a large area of the external Galactic bulge is also covered.

After collecting a large sample of photometric data (over 100 epochs), spanning three observing seasons, the region of the Galactic disk with $190<l<345$ degrees is ready for exploration. Here we present the preliminary report on the first quick search for Cepheids and RR Lyrae stars in these fields of the OGLE GVS.

\section{Observations}

The OGLE-IV survey has been conducted using the 1.3-m Warsaw telescope with a 32 CCD detector wide field mosaic camera located at the Las Campanas Observatory, Chile. The field of view of the OGLE-IV camera is 1.4 square degrees with the pixel scale of 0.26 arsec/pixel. The OGLE GVS has been started in February 2013 and it is still conducted albeit with different cadence and priority of fields compared to previous seasons. The main variability survey is a shallow one with the exposure

^udalski@astrouw.edu.pl 

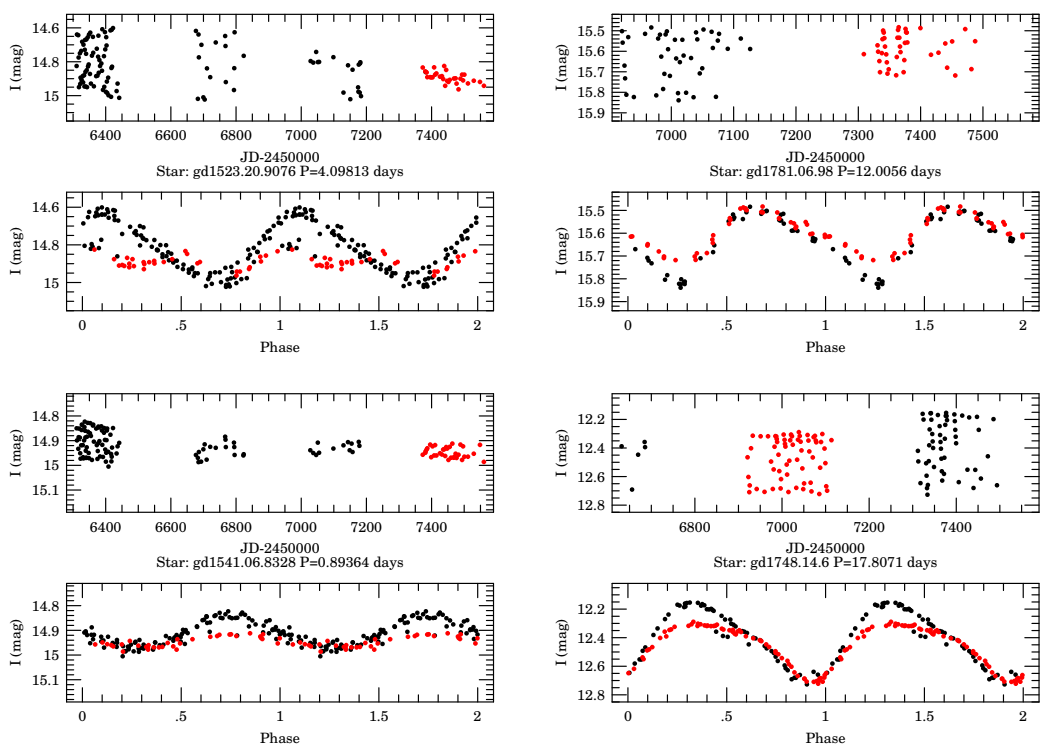

Figure 1. Example light curves of variable stars mimicking pulsating-like variability behavior. Red points mark observing season with considerably different shape of the light curve.

time of 25 seconds. Most observations are collected in the $I$-band filter for variability search. A total of 120-150 epochs in each of the fields presented here have been collected so far. Also, about ten or more observations have been secured in the $V$-filter for color information.

The collected images have been reduced with the OGLE standard photometric pipeline which is based on the image subtraction technique - DIA ([10]). The faint limit for variability search in the OGLE GVS is about $I \approx 18.5 \mathrm{mag}$.

\section{Cepheids and RR Lyrae stars from the OGLE GVS}

We carried out the pilot search for pulsating variables of Cepheid and RR Lyrae types among about 560 million stellar sources detected on the OGLE GVS images of the Galactic plane. This "fast search" is different than the typical global OGLE variable stars searches. It has been conducted on pre-selected subsample of stars - only those which displayed clear variability signatures on the difference images. Minimum 15 detections of variable flux in the difference images were required to include a star to this sample.

The pre-selected variable star candidates were then subject to period search. Two techniques were used: analysis of variance, AoV ([5]), and Fourier analysis implemented as FNPEAKs program ([3]). Light curves of objects with significant periodic signals were further investigated. First, their shape was fitted with Fourier series and the Fourier parameters $R_{21}$ and $\phi_{21}$ were calculated. In the next step of pre-selection, only candidates with Fourier parameters in a wide range covering typical regions occupied by Cepheids and RR Lyrae stars on the $R_{21}$ vs. $\log P$ and $\phi_{21}$ vs. $\log P$ diagrams were left. Finally, the candidates were visually inspected and objects with light curves of non-pulsating origin (e.g., eclipsing binaries) were removed from the ultimate list of Cepheid/RR Lyrae candidates. 

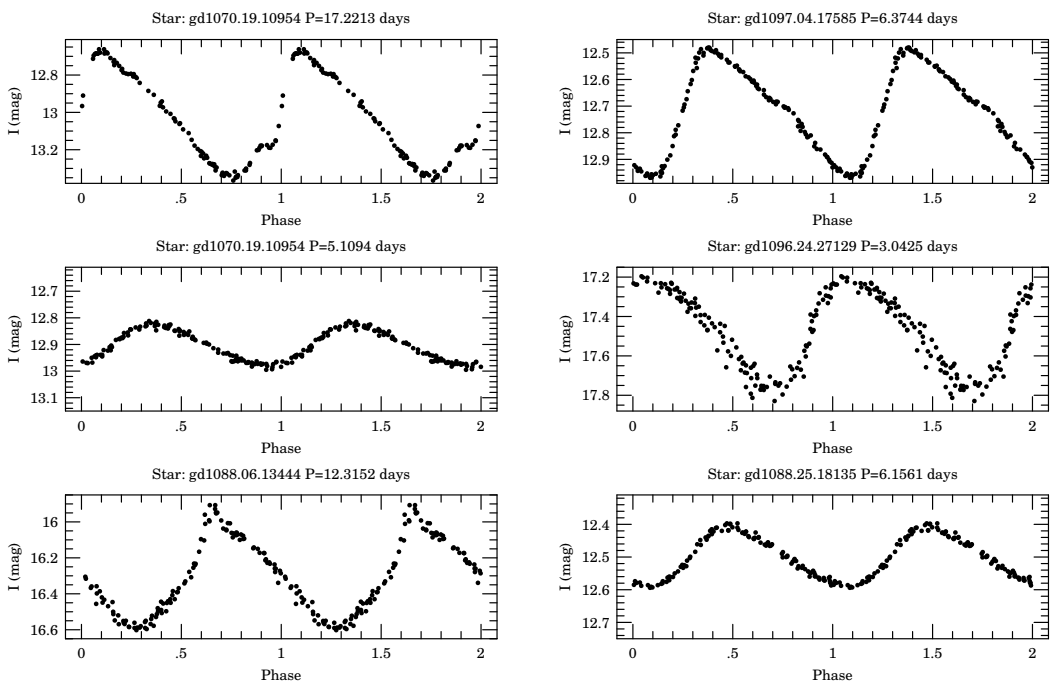

Figure 2. Example light curves of newly discovered Galactic disk Cepheids.

Surprisingly, the list of objects at this stage was still severely contaminated. The main source of fake identifications were spotted stars which can mimic very well the light curve of pulsating star. On the other hand the shape of the variability in such stars evolves with time and in the case of long-term monitoring, as during OGLE GVS, spotted stars can be extracted and separated from genuine pulsators. Figure 1 presents light curves of a few examples of spotted stars mimicking in some observing seasons pulsating stars.

After visual inspection of the OGLE Cepheid/RR Lyrae candidates, 1930 and 2170 objects were left as very likely Galactic Cepheids and RR Lyrae type stars, respectively. At this stage of analysis the boundary between these two groups was set artificially at $P=0.7$ day. Figure 2 presents a small gallery of newly discovered Galactic Cepheids. The list of Cepheids contains classical Cepheids as well as type II objects.

Preliminary lists of the OGLE Cepheid and RR Lyrae candidates will additionally be refined when the color data are available. Nevertheless, the purity of the sample is already high. The completeness of these samples is different. While the Galactic Cepheids are bright enough to be detected on the OGLE subtracted images, fainter RR Lyrae stars located farther in the halo are certainly missed in our preselected sample of analyzed stars. We expect that many additional fainter RR Lyrae stars will be detected during the final exensive search for variables in the OGLE GVS fields.

Figures 3 and 4 show the distribution of new OGLE Galactic Cepheids and RR Lyrae stars on the map of the sky, respectively. Figures 5 and 6 present distribution of these groups of stars in the real sky. Even without deeper analysis one can easily notice differences in distributions. For example, while RR Lyrae stars are generally located symmetrically around the Galactic plane, Cepheids lie mostly below the plane over a long range of observed Galactic longitudes.

The new OGLE samples of Galactic Cepheids and RR Lyrae stars will be released to the community in the next months via the OCVS. They will constitute the next huge OGLE datasets of these important pulsating stars. 


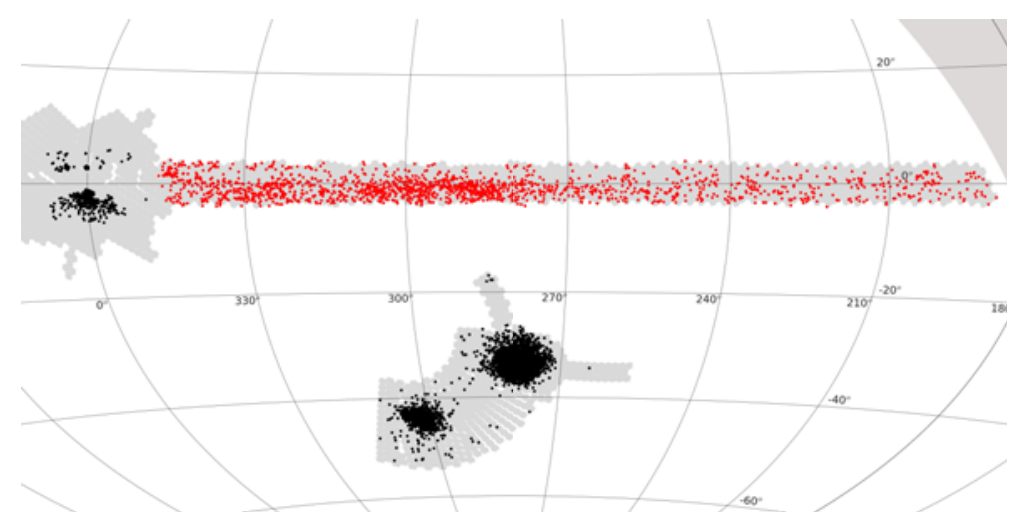

Figure 3. OGLE Cepheids in the sky. The sky map is in the Galactic coordinates $(l, b)$. Light gray shaded areas show the regions regularly monitored by the OGLE-IV survey. Dark gray shaded region in the upper right corner indicates part of the sky not accessible from Las Campanas Observatory. Red dots present Cepheid star candidates found in the OGLE GVS fields. Black dots mark positions of the OGLE Cepheids in other OGLE-IV targets.

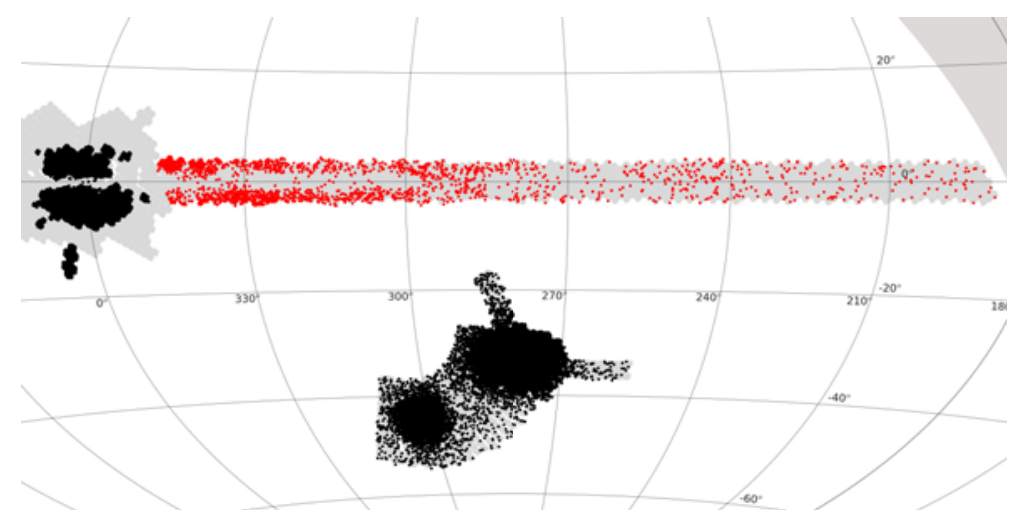

Figure 4. OGLE RR Lyrae stars in the sky. The sky map is in the Galactic coordinates $(l, b)$. Light gray shaded areas show the regions regularly monitored by the OGLE-IV survey. Dark gray shaded region in the upper right corner indicates part of the sky not accessible from Las Campanas Observatory. Red dots present RR Lyrae star candidates found in the OGLE GVS fields. Black dots mark positions of the OGLE RR Lyrae stars in other OGLE targets.

Acknowledgments: The OGLE project has received funding from the National Science Centre, Poland, grant MAESTRO 2014/14/A/ST9/00121.

\section{References}

[1] Jacyszyn-Dobrzeniecka, A.M., Skowron, D.M., Mróz, P., et al., Acta Astron., 66, 149 (2016)

[2] Jacyszyn-Dobrzeniecka, A.M., Skowron, D.M., Mróz, P., et al., Acta Aston., 67, 1 (2017)

[3] Kołaczkowski, Z., http://helas. astro.uni .wroc.pl/deliverables.php?lang=en\&active=fnpeaks (2016) 


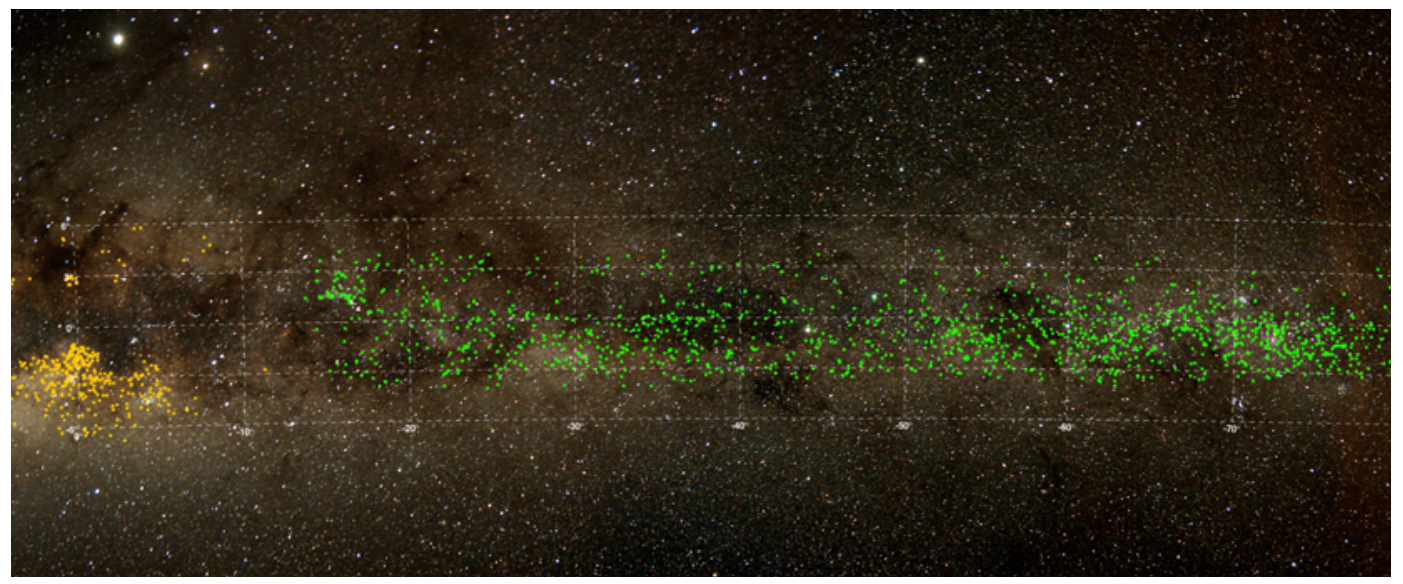

Figure 5. Details of the distribution of OGLE Cepheids in the Galactic plane. Green dots indicate positions of the OGLE-IV Cepheid candidates from the OGLE GVS. Yellow dots are OGLE Cepheids from the Galactic bulge fields.

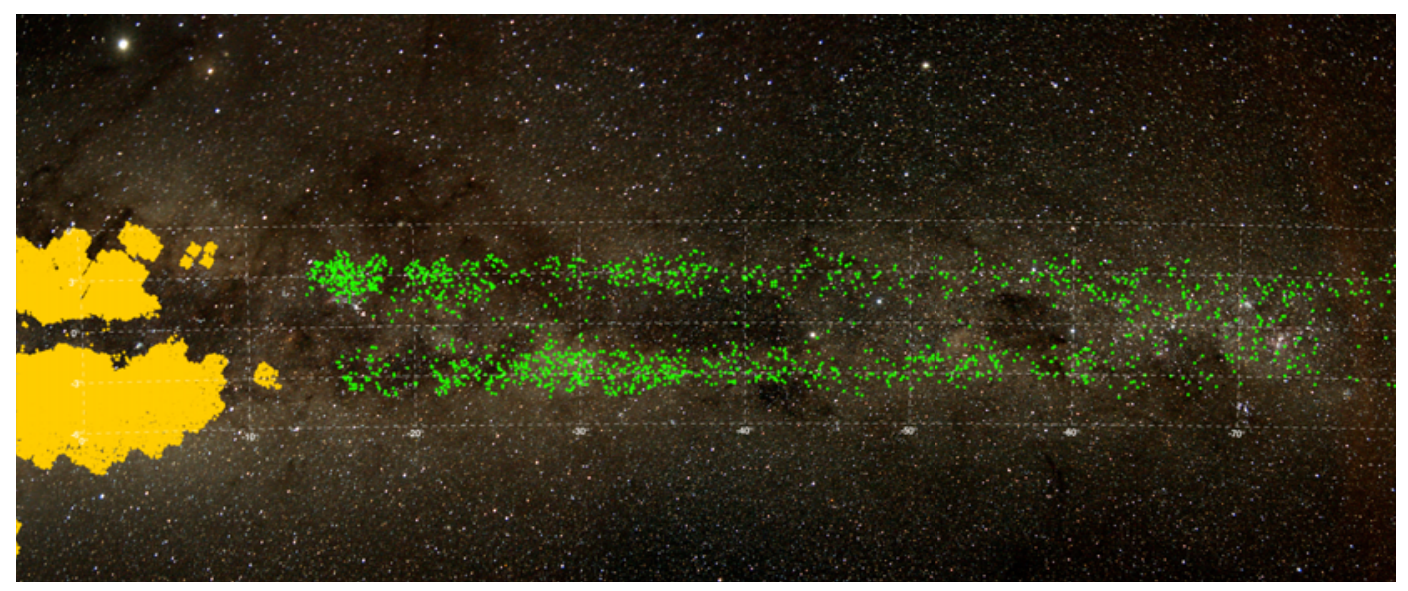

Figure 6. Details of the distribution of OGLE RR Lyrae stars in the Galactic plane. Green dots indicate positions of the OGLE-IV RR Lyrae star candidates from the OGLE GVS. Yellow dots are OGLE RR Lyrae stars from the Galactic bulge fields.

[4] Pietrukowicz, P., Kozłowski, S., Skowron, J., et al., ApJ, 811, 113 (2015)

[5] Schwarzenberg-Czerny, A., MNRAS, 241, 153 (1989)

[6] Soszyński, I., Udalski, A., Szymański, M.K., et al., Acta Astron., 64, 177 (2014)

[7] Soszyński, I., Udalski, A., Szymański, M.K., et al., Acta Astron., 65, 297 (2015)

[8] Soszyński, I., Udalski, A., Szymański, M.K., et al., Acta Astron., 66, 131 (2016)

[9] Soszyński, I., Pawlak, M., Pietrukowicz, P., et al., Acta Astron., 66, 405 (2016)

[10] Udalski, A., Szymański, M.K., \& Szymański, G., Acta Astron., 65, 1 (2015) 